\title{
White Fibrous Papulosis of the Neck Treated With Fractionated 1550-nm Erbium Glass Laser: A Case Report
}

\author{
Suparuj Lueangarun ${ }^{1}$, Ratchathorn Panchaprateep ${ }^{2 *}$ \\ 'Division of Dermatology, Chulabhorn International College of Medicine, Thammasat University, Pathum Thani, Thailand \\ ${ }^{2}$ Division of Dermatology, Department of Medicine, Faculty of Medicine, King Chulalongkorn Memorial Hospital, Bangkok, \\ Thailand
}

\author{
*Correspondence to \\ Ratchathorn Panchaprateep, MD, \\ PhD; Division of Dermatology, \\ Department of Medicine, Faculty \\ of Medicine, King Chulalongkorn \\ Memorial Hospital, Bangkok, \\ Thailand 10330 . \\ Tel: +66898923819; \\ Email:nim_bonus@hotmail.com \\ Published online 27 October 2016
}

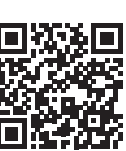

\begin{abstract}
Introduction: Despite its clinical features of multiple, confluent, small, whitish, smooth, and clear-demarcate papules on the neck and back, the pathogenesis of white fibrous papulosis of the neck (WFPN) is still unknown. The lesions increase progressively and do not regress over time. However, no effective treatment has yet been identified.

Case Report: We reported the successful results of a female patient receiving efficacious treatment for her extensive lesions of WFPN with nonablative fractional photothermolysis laser (Fractionated 1550-Erbium Glass laser).

Conclusion: This photothermolysis laser could then be suggestive as the therapeutic option for WFPN.

Keywords: Papulosis; Laser; Treatment; White fibrous papulosis of the neck; Nonablative fractional photothermolysis laser; Fractionated 1550-Erbium Glass laser.
\end{abstract}

\begin{abstract}
Introduction
White fibrous papulosis of the neck (WFPN) is generally characterized by multiple, confluent, small, clearly demarcated, whitish papules on the neck and back. Other anatomical areas involved have also been reported, such as upper sternum, nape, and upper arm. Moreover, the papules are asymptomatic and not attached to hair follicles.

WFPN is most commonly reported in patients with a mean age of 60 years, and at the youngest age of 28 years. In spite of its non-specially defined racial and gender prevalence, it usually occurs among Japanese males and rarely in European females. ${ }^{1}$ Though mostly found with progressively increased lesions and not regressive over time, the pathogenesis of WFPN remains unknown. Nonetheless, several ultrastructural studies suggest that age- or sun-related damages could be indicative factors for WFPN. ${ }^{2}$ Particularly, most patients are noted without history of prolonged sun exposure or similar lesions in the same family. More importantly, no effective treatments for WFPN have been established.
\end{abstract}

\section{Case Report}

A 26-year-old female presented with 1-year history of gradually increased number of asymptomatic whitish small papules on the neck, upper chest wall, and upper back. Physical examination revealed multiple, round to oval, smooth surfaced, 2- to 3-mm discrete whitish papules on the anterior neck, shoulders, upper chest wall, and upper back, yet unrelated to hair follicles. Meanwhile, dermoscopic examination demonstrated well-circumscribed, homogeneous, white areas, and short, thin vessels, without follicular involvement. Besides, a dome-shaped portion of skin with sparse superficial perivascular infiltrate was noted by histopathology. Furthermore, fragments of elastic fibers decreased in diameters and numbers at the center of lesions were evident from elastic stains (Figure $1 \mathrm{~A}$ and $1 \mathrm{~B}$ ); whilst alcian blue stains showed no mucins. Hence, the diagnosis of WFPN was confirmed.

Nonablative fractional laser (NAFL) is currently approved by the US Food and Drug Administration (FDA) for the treatment of various skin disorders, such as photoaging, periorbital rhytides, pigmented lesions, melasma, soft tissue coagulation, acne and surgical scars, as well as precancerous skin disorders like actinic keratosis. ${ }^{3}$ Thus, we postulated that NAFL may also be effective on WFPN. Fractional 1550-nm Ytterbium/Erbium fiber laser (Fines$\operatorname{can}^{\circledR}$; TNC Medditron Co., Ltd., Thailand) using 7-cm tip, with the same recommended parameter for acne scar of $30 \mathrm{~mJ} / \mathrm{cm}^{2}$ and 600 microscopic treatment zones (MTZ)/ $\mathrm{cm}^{2}\left(200 \mathrm{MTZ} / \mathrm{cm}^{2}\right.$ per pass for 3 passes $)$ and $10 \%$ coverage with the endpoint of mild erythema, was performed

Please cite this article as follows: Lueangarun S, Panchaprateep R. White fibrous papulosis of the neck treated with fractionated 1550-nm erbium glass laser: a case report. J Lasers Med Sci. 2016;7(4):256-258. doi:10.15171/jlms.2016.45. 
on the testing area of $100 \mathrm{~cm}^{2}$ along the right chest below the clavicle every 2 weeks for 8 sessions. The patient was anaesthetized using topical lidocaine $2.5 \%$ and prilocaine $2.5 \%$ cream under occlusion for 45 minutes. Cold air was also applied during treatment to lessen the pain. Then, ice packs were placed at the treated area for 20 minutes after the treatment. Following the 8 sessions of laser treatment, significant clinical improvement was observed. The evaluation of treatment results by 3-blinded dermatologists and the patient herself similarly showed 50\%-75\% and $60 \%-70 \%$ improvement, respectively. The pain was also tolerable with visual analog scale of 2 out of 10 . The persistence of treatment results was finally noted after 16 months of follow-up (Figure 2A and 2B).

\section{Discussion}

Despite its benign nature, WFPN is cosmetically undesirable and sometimes itchy. ${ }^{4}$ For this reason, many medical and surgical treatments have been applicably implemented and performed with variable results. With some topical treatments like topical tretinoin and antioxidant (to inhibit intrinsic free-radical induced aging), the improvement was not notable. Hence, surgical excision should be an alternative choice of treatment in several cases with circumscribed lesions. ${ }^{5}$

Through the induction of microthermal zones of injury, fractional laser has been reported to improve photoaged skin conditions, such as facial rhytids, dyspigmentation,
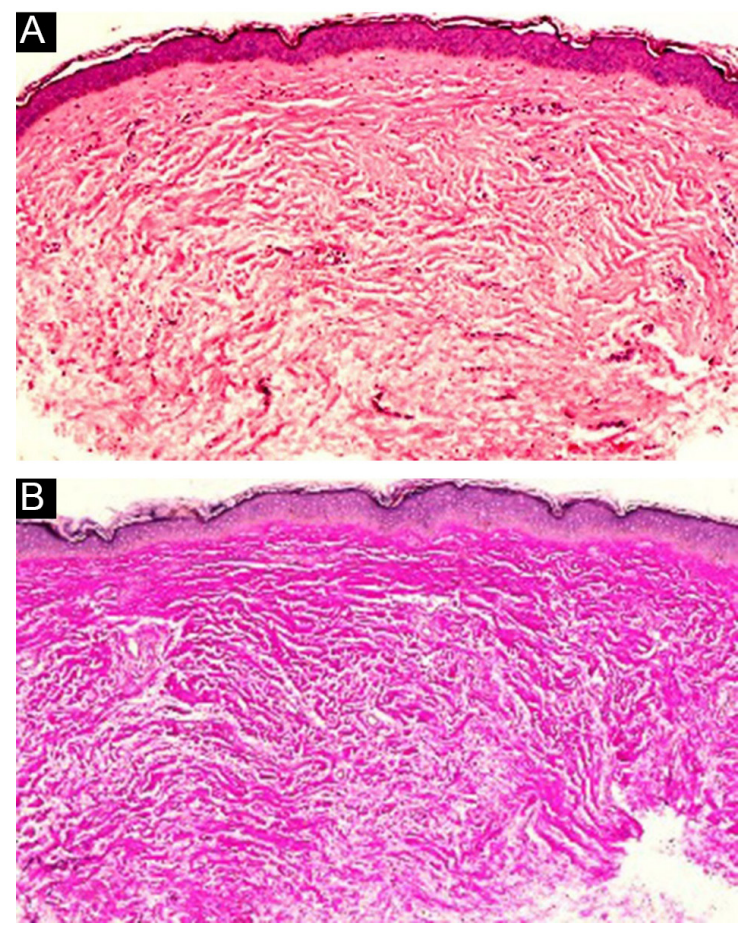

Figure 1. (A) Histological findings of lesions before treatment showed slightly elevated dermal nodule composed of slightly thickened collagen fibers and dilated capillaries (20x, hematoxylin and eosin). (B) Marked decrease of thin and fragmented elastic fibers (20x, elastic stain).
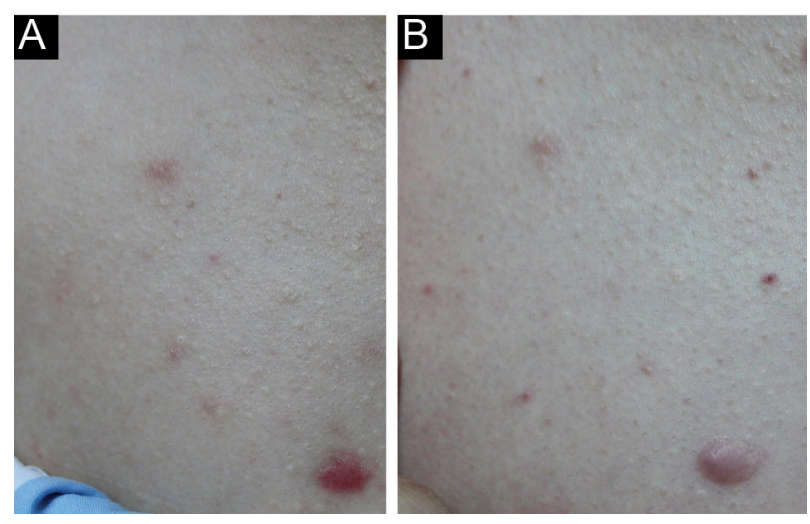

Figure 2. (A) Multiple, 2-3 mm, white, round papules were located on chest wall. (B) The results at 16 months after 8 laser treatments revealed decreasing in numbers and sizes of the lesions.

tissue laxity and skin roughness, as well as poikiloderma of Civatte and pigmentary skin disorders. ${ }^{67}$ Histopathological examination revealed that the hyalinized collagen in the Grenz zone, linked to photoaging, was replaced by newly organized collagen fibers, suggesting neocollagenogenesis. Besides, the elastic fibers were better arranged and denser, with increased expression for up to one year after three laser treatments. ${ }^{8,9}$

Since WFPN is arguably a feature of photoaging or intrinsic aging, with characteristically superficial dermal fibrosis of scant elastolysis, it is presumed that fractional non-ablative laser can be a suitable alternative for this disease entity. The remodeled fine elastic fibers and the increased collagen deposition in the upper dermis, therefore, correlated with the clinical improvement of smaller and smother lesions. In particular, there is histological evidence from previous studies that NAFL is likely to induce the transepidermal elimination of the dermal content, such as the elastic fiber fragments through these multiple small channels created by the laser.

In conclusion, a female patient is reported with successful and efficacious treatment for her extensive lesions of WFPN, using NAFL. The treatment could be considered as an efficient and safe therapeutic option for WFPN.

\section{Ethical Considerations}

The informed consent was obtained from patient prior to each procedure.

\section{Conflict of Interests}

None to be declared.

\section{References}

1. Song $\mathrm{YC}, \mathrm{Oh} \mathrm{BH}, \mathrm{Ko} \mathrm{JH}$, et al. A case of fibroelastolytic papulosis on the neck of a young man. Ann Dermatol. 2011;23(2):193-197. doi:10.5021/ad.2011.23.2.193.

2. Zanca A, Contri MB, Carnevali C, Bertazzoni MG. White fibrous papulosis of the neck. Int $J$ Dermatol. 1996;35(10):720-722.

3. Tierney EP, Kouba DJ, Hanke CW. Review of fractional photothermolysis: treatment indications and efficacy. 
Dermatol Surg. 2009;35(10):1445-1461. doi:10.1111/j.15244725.2009.01258.x.

4. Wang DG, Zhu WY. White fibrous papulosis of the neck: a chinese case. J Dermatol. 2005;32(6):497-499.

5. Gencoglan G, Ceylan C, Kazandi AC. White fibrous papulosis of the neck. Cutan Ocul Toxicol. 2011;30(1):6971. doi:10.3109/15569527.2010.521223.

6. Behroozan DS, Goldberg LH, Glaich AS, Dai T, Friedman PM. Fractional photothermolysis for treatment of poikiloderma of civatte. Dermatol Surg. 2006;32(2):298301.

7. Bogdan Allemann I, Kaufman J. Fractional photothermolysis
-- an update. Lasers Med Sci. 2010;25(1):137-144. doi:10.1007/ s10103-009-0734-8.

8. Borges J, Cuzzi T, Mandarim-de-Lacerda CA, ManelaAzulay M. Fractional Erbium laser in the treatment of photoaging: randomized comparative, clinical and histopathological study of ablative $(2940 \mathrm{~nm})$ vs. nonablative $(1540 \mathrm{~nm})$ methods after 3 months. An Bras Dermatol. 2014;89(2):250-258.

9. Fournier N, Dahan S, Barneon G, et al. Nonablative remodeling: clinical, histologic, ultrasound imaging, and profilometric evaluation of a $1540 \mathrm{~nm}$ Er:glass laser. Dermatol Surg. 2001;27(9):799-806. 